\title{
Selection, application and monitoring of Lactobacillus paracasei strains as adjunct cultures in the production of Gouda-type cheeses
}

\author{
Koenraad Van Hoorde ${ }^{\mathrm{a}, \mathrm{b}, *}$, Isabelle Van Leuven ${ }^{\mathrm{c}}$, Patrick Dirinck ${ }^{\mathrm{c}}$, Marc Heyndrickx ${ }^{\mathrm{d}}$, Kathleen Coudijzer ${ }^{\mathrm{d}}$, \\ Peter Vandamme ${ }^{\text {a }}$, Geert Huys ${ }^{\text {a,e }}$ \\ a Laboratory of Microbiology, Ghent University, K.L. Ledeganckstraat 35, B-9000 Gent, Belgium \\ ${ }^{\mathrm{b}}$ Faculty of Applied Engineering Sciences, University College Ghent, Campus Schoonmeersen, Schoonmeersstraat 52, B-9000 Gent, Belgium \\ ' Laboratory for Flavour Research, Catholic University College Ghent, K.U. Leuven Association, Gebr. Desmetstraat 1, B-9000 Gent, Belgium \\ ' Unit Technology and Food, Institute for Agricultural and Fisheries Research (ILVO), Brusselsesteenweg 370, B-9090 Melle, Belgium \\ e BCCM/LMG Bacteria Collection, Ghent University, K.L. Ledeganckstraat 35, B-9000 Gent, Belgium
}

\section{A R T I C L E I N F O}

\section{Article history:}

Received 9 December 2009

Recieved in revised form 20 April 2010

Accepted 11 May 2010

\section{Keywords}

Adjunct

Gouda cheese

Lactobacillus paracasei

DGGE

GC-MS

Sensory analysis

\begin{abstract}
A B S T R A C T
Raw milk cheeses have more intense flavours than cheeses made from pasteurized milk and harbour strains with potential adjunct properties. Two Lactobacillus paracasei strains, R-40926 and R-40937, were selected as potential adjunct cultures from a total of 734 isolates from good quality artisan raw milk Gouda-type cheeses on the basis of their prevalence in different cheese types and/or over several production batches, safety and technological parameters. Conventional culturing, isolation and identification and a combined PCR-DGGE approach using total cheese DNA extracts and DNA extracts obtained from culturable fractions were employed to monitor viability of the introduced adjuncts and their effect on the cheese microbiota. The control cheese made without adjuncts was dominated by members of the starter, i.e. Lactococcus lactis and Leuconostoc pseudomesenteroides. In the cheeses containing either R-40926 or R-40937, the respective adjuncts increased in number as ripening progressed indicating that both strains are well adapted to the cheese environment and can survive in a competitive environment in the presence of a commercial starter culture. Principal component analysis of cheese volatiles determined by steam distillation-extraction and gas chromatography-mass spectrometry could differentiate cheeses made with different concentrations of adjunct R-40926 from the control cheese, and these differences could be correlated to the proteolytic and lipolytic properties of this strain. Collectively, results from microbiological and metabolic analyses indicate that the screening procedure followed throughout this study was successful in delivering potential adjunct candidates to enrich or extend the flavour palette of artisan Gouda-type cheeses under more controlled conditions.
\end{abstract}

(c) 2010 Elsevier B.V. All rights reserved.

\section{Introduction}

Flavour formation during cheese ripening is the result of a series of complex chemical and biochemical conversions of milk components (Smit et al., 2005). Essentially, the typical cheese flavour arises from three main processes i.e. proteolysis of milk caseins, lipolysis of milk fat and fermentation of (residual) lactose, lactate and citrate which collectively result in the production of a complex mixture of volatile aroma and non-volatile taste compounds (Marilley and Casey, 2004; Smit et al., 2005). Next to the action of enzymes from rennet and milk, microbial enzyme activities are largely responsible for the formation of flavour compounds during cheese ripening. For Gouda-type cheeses, micro-organisms involved in flavour development basically can be divided in two major groups, i.e. starter and non-starter lactic

\footnotetext{
* Corresponding author. Laboratory of Microbiology, Ghent University, K.L. Ledeganckstraat 35, B-9000 Gent, Belgium. Tel.: +320 92645130.

E-mail address: koenraad.vanhoorde@ugent.be (K. Van Hoorde).
}

acid bacteria ( $\mathrm{LAB}$ ). Although the main role of the starter culture is the production of lactic acid, it also largely contributes to proteolysis and lipolysis and hence flavour formation (Kieronczyk et al., 2003; Smit et al., 2005). Non-starter lactic acid bacteria (NSLAB), which may enter the cheese making process through the milk, the cheese making equipment and/or the production environment and which gradually become the dominant microbiota during ripening reaching numbers up to $10^{7}-10^{8} \mathrm{cfu} / \mathrm{g}$, also play an important role in the development of the organoleptic properties of mature cheese as a result of lipid and protein metabolism (Fox et al., 1998). As most LAB produce a range of proteinases, peptidases and amino acid converting enzymes, the proteolytic pathway is by far the most important process during flavour formation (McSweeney, 2004). In contrast, only moderate to little lipolysis occurs in Gouda and many other cheese types (Collins et al., 2003; McSweeney, 2004) probably due to a restricted or lack of lipolytic activity of most LAB (Broadbent and Steele, 2005).

Over the years, large-scale cheese production, the need for process and quality control, the application of a limited number of starter 
cultures exhibiting similar functional properties and the increased use of pasteurization processes intended to reduce the microbiological load of milk have resulted in a higher uniformity of many industrial cheeses (Wouters et al., 2002). In parallel to product consistency and quality, however, there is also a growing need for product diversification on the cheese market. To this end, the use of bacterial strains with attractive flavour-forming abilities is one of the possible strategies to answer to the increasing demand for cheeses with new or improved organoleptic properties. The much more diverse and heterogeneous microbiota and the more intense and stronger flavour of raw milk cheeses strongly suggest that NSLAB play a significant role during the ripening process and that their presence is beneficial for developing a full-flavoured cheese (Buchin et al., 1998; Grappin and Beuvier, 1997). Hence, raw milk cheeses can be an important source of adjunct cultures and the use of such adjuncts has already been investigated in several studies (Antonsson et al., 2003; Gómez-Ruiz et al., 2008; Lynch et al., 1996). On the other hand, the addition of adjunct cultures may also give rise to off-flavours or quality defects (Herreros et al., 2007; Lee et al., 1990), which highlights the relevance of careful screening of potential adjunct strains before their introduction in large-scale model fermentations.

The aim of the present study was to evaluate a method to screen for potentially interesting $\mathrm{LAB}$ adjunct strains by determining the impact of two selected Lactobacillus paracasei strains on the microbiological and volatile composition of a Gouda-type model cheese. The potential effects of the cheese making process on growth, viability and survival of the introduced adjunct strains and the influence of the adjunct on the cheese microbiota were assessed using conventional culturing, identification and typing and PCR-DGGE analysis of total cheese DNA extracts and DNA extracts obtained from culturable LAB fractions. In parallel, steam distillation-extraction and gas chromatography-mass spectrometry (SDE-GC-MS) were employed to detect possible differences in the sensory profile between the control cheese and the adjunct-containing cheeses.

\section{Material and methods}

\subsection{Initial selection of potential adjunct cultures}

In the course of a previous study, the microbial diversity associated with the production and ripening of two Flemish artisan raw milk Gouda-type cheeses produced at the same location was assessed during two independent sampling campaigns using selective isolations and polyphasic identification (Van Hoorde et al., 2008b). Dereplication of the initial set of 734 isolates by (GTG) $)_{5}$-PCR fingerprinting resulted in a subset of 298 genotypically unique isolates. The majority of these isolates $(n=244)$ grouped in 25 genotypic clusters, whereas the other 54 isolates remained unclustered and belonged to species representing less than $1 \%$ of the total number of isolates. From clusters that contained isolates recovered from both cheeses and/or recovered during both sampling campaigns, two to three isolates were selected for genotyping using amplified fragment length polymorphism (AFLP) analysis as previously described (Van Hoorde et al., 2008a). Based on their unique AFLP fingerprints, a set of 19 mesophilic strains comprising L. paracasei $(n=10)$, Pediococcus pentosaceus $(n=4)$ and single strains of Lactobacillus brevis, Lactobacillus curvatus, Lactobacillus perolens, Lactobacillus rhamnosus and Lactobacillus plantarum was used for further taxonomic characterization, antibiotic susceptibility testing and functional screening.

\subsection{Antibiotic susceptibility testing}

Selected strains were screened for phenotypic resistance to erythromycin and tetracycline, i.e. the two most commonly found resistance phenotypes in non-enterococcal LAB (Klare et al., 2007; Van Hoek et al., 2008), using a modified version of the Kirby-Bauer disk diffusion method (Bauer et al., 1966). For this purpose, strains were grown on the LAB Susceptibility test Medium (LSM) agar formulation (Klare et al., 2005) consisting of 90\% Isosensitest broth (CM0473, OXOID), 10\% MRS broth (CM0359, OXOID, Basingstoke, UK) and $10 \mathrm{~g} / \mathrm{L}$ General Purpose Agar $\mathrm{N}^{\circ} 2$, (LAB M, Lancashire, UK). Prior to testing, isolates were subcultured in $5 \mathrm{~mL}$ MRS broth (CM0359, OXOID) for $24 \mathrm{~h}$ at $28{ }^{\circ} \mathrm{C}$ under anaerobic conditions. Subsequently, $100 \mu \mathrm{L}$ of the broth culture was inoculated on LSM agar and grown overnight. For preparation of the inoculum, colonies were suspended in sterile saline solution $(0.85 \% \mathrm{w} / \mathrm{v} \mathrm{NaCl})$ until an $\mathrm{OD}_{590}$ equivalent of McFarland (McF) standard 1 was obtained. LSM agar plates were inoculated with the standardized McF 1 inoculum and paper disks (OXOID) impregnated with erythromycin (15 $\mu \mathrm{g}$; CT0020) and tetracycline (30 $\mu \mathrm{g}$; СТ0054) were applied to the inoculated plates. Following anaerobic incubation for $48 \mathrm{~h}$ at $28^{\circ} \mathrm{C}$, inhibition zone diameters were measured using a digital calliper (Mauser Digital 2, Ludwigsburg, Germany). Interpretation of results to discriminate between resistant and susceptible phenotypes was based on the guidelines proposed by Charteris et al. (1998).

Strains displaying tetracycline resistance in disk diffusion were subsequently subjected to MIC determination as described previously (Masco et al., 2006) using LSM broth as inoculation medium. Strains for which phenotypic tetracycline resistance was confirmed were screened by PCR for the presence of tetracycline resistance genes tet( $\mathrm{M})$, tet $(\mathrm{W})$, tet $(\mathrm{O})$ and tet $(\mathrm{T})$ using the $\mathrm{PCR}$ primers and conditions as described by Masco et al. (2006).

\subsection{Assessment of proteolytic and lipolytic properties}

Protease and lipase activities were assessed on two differential media, milk plate count agar (MPCA) (CM0681, OXOID) and tributyrin agar (TA) (PM0004, OXOID, Basingstoke, UK), respectively. Isolates to be tested for the presence of protease and/or lipase activity were first recovered from MicroBank ${ }^{\mathrm{TM}}$ vials by incubating them aerobically in MRS broth for $24-48 \mathrm{~h}$ at $28^{\circ} \mathrm{C}$, after which $10 \mu \mathrm{L}$ of the grown culture was plated on MRS agar and again incubated for $24-48 \mathrm{~h}$ at $28^{\circ} \mathrm{C}$ under aerobic conditions. Subsequently, MPCA and TA plates were inoculated in duplicate and incubated under aerobic conditions at $14^{\circ} \mathrm{C}$, the temperature of ripening, and $28^{\circ} \mathrm{C}$, the optimal growth temperature of both isolates. Conversion of casein by proteolytic activity on MPCA plates resulted in the formation of clear zones around the grown colonies. Lipolytic activity was recognized through the formation of clear halos around colonies on TA plates resulting from hydrolysis of the ester bonds in the triglyceride tributyrin and the subsequent release of butyric acid.

\subsection{Preparation of adjunct cultures and manufacturing of model cheeses}

Following recovery in MRS broth, subcultures of the two selected L. paracasei adjunct strains were transferred onto MRS agar and incubated aerobically at $28{ }^{\circ} \mathrm{C}$. After $48 \mathrm{~h}$, cells were harvested and suspended in sterile $0.85 \% \mathrm{w} / \mathrm{v}$ saline solution to obtain concentrations of $10^{8} \mathrm{cfu} / \mathrm{mL}$ or $10^{10} \mathrm{cfu} / \mathrm{mL}$. These suspensions were used to inoculate $100 \mathrm{~L}$ of cheese milk to obtain a final adjunct concentration of $10^{3}$ or $10^{5} \mathrm{cfu} / \mathrm{mL}$.

Model Gouda-type cheeses were made in a pilot plant from pasteurized ( $15 \mathrm{~s}$ at $72{ }^{\circ} \mathrm{C}$ ) milk using standard technology for the manufacturing of Gouda cheese (van den Berg, 2003). In total, four cheeses were produced on the same day from the same batch of pasteurized milk using the same basic ingredients to ensure that the respective cheeses only differed in the presence or absence of an adjunct culture. These four cheeses included a control cheese (i.e. G_C) without adjunct culture, a cheese with adjunct culture R-40937 in a final concentration of $10^{5} \mathrm{cfu} / \mathrm{mL}$ (i.e. G_40937), and two cheeses made with adjunct culture R-40926 in a final concentration of $10^{3}$ and $10^{5} \mathrm{cfu} / \mathrm{mL}$ (i.e. G_40926(3) and G40926(5), respectively). Before adding starter and adjunct cultures, the temperature of the milk was brought to $30^{\circ} \mathrm{C}$. 
$\mathrm{CaCl}_{2}(5 \mathrm{~g} / 100 \mathrm{~L})$ and saltpetre $\left(15 \mathrm{~g} \mathrm{NaNO}_{3} / 100 \mathrm{~L}\right)$ were added under constant stirring after 10 and 20 min, respectively. After addition of the rennet $(30 \mathrm{~mL}$ of a $1: 10,000$ strength $520 \mathrm{mg}$ chymosin/100 mL rennet solution), the cheese milk was put at rest for approximately 30 min of renneting and the resulting coagulum was cut for 20 to $25 \mathrm{~min}$. After cutting, scalding was performed by which approximately half of the volume of the whey was expelled and replaced with warm $\left(40{ }^{\circ} \mathrm{C}\right.$ ) water followed by $20 \mathrm{~min}$ of stirring at $35^{\circ} \mathrm{C}$, removal of the whey, pre-pressing ( $5 \mathrm{~min}$ ), moulding of rectangular cheeses of approximately $2.5 \mathrm{~kg}$ and pressing $(3 \mathrm{~h})$. Cheeses were brine salted for $15 \mathrm{~h}$. Ripening temperature of the cheeses was $14^{\circ} \mathrm{C}$.

\subsection{Sampling and bacteriological analysis}

The cheese production process was monitored by collecting samples from milk (M), starter (S), curd (C), one-day (D1) and one-week (W1) old cheeses and cheeses ripened for 3 and 5 weeks (W3 and W5, respectively).

For bacteriological processing, milk ( $1 \mathrm{~mL}$ ) and cheese $(1 \mathrm{~g})$ samples were homogenized in $9 \mathrm{~mL} 2 \% \mathrm{w} / \mathrm{v}$ trisodium citrate using a stomacher lab-blender, and 10-fold serial dilutions were prepared in peptone physiological solution $(0.1 \% \mathrm{w} / \mathrm{v}$ bacteriological peptone (L37, OXOID) and $0.85 \% \mathrm{w} / \mathrm{v} \mathrm{NaCl}$ ). A volume of $0.1 \mathrm{~mL}$ of the appropriate dilutions was spread plated in triplicate on three media supporting the growth of various groups of LAB: Kanamycin Aesculin Azide Agar Base (KAAAB) (CM0591, OXOID) supplemented with $20 \mathrm{mg} / \mathrm{L}$ kanamycin (SR00925, OXOID) for enterococci, M17 agar (CM0785, OXOID) supplemented with lactose $(0.5 \%)$ for lactococci and Mayeux, Sandine, Elliker agar (MSE) (401738, Biolife, Milan, Italy) for leuconostocs. These three media also support growth of lactobacilli (Van Hoorde et al., 2008b) and of the two selected adjunct cultures R-40926 and R-40937. KAAA and LM17 plates were incubated for $48-72 \mathrm{~h}$ at $37{ }^{\circ} \mathrm{C}$ under aerobic conditions whereas MSE agar plates were aerobically incubated for 5-7 days at $28{ }^{\circ} \mathrm{C}$. For the four cheeses, a total of 1147 isolates were randomly selected from plates containing 30-300 cfu.

In addition to colony selection, bulk cell suspensions for DGGE analyses (Ercolini et al., 2001) were prepared for each medium by suspending the culturable fractions of the plated $10^{-1}$ milk and cheese sample dilutions in $1.0 \mathrm{~mL} 1 \times$ TE buffer ( $10 \mathrm{mM}$ Tris base, $1 \mathrm{mM}$ EDTA).

\subsection{DNA extraction from pure cultures, culturable fractions and total cheese DNA}

DNA extraction from pure cultures and bulk cell suspensions was performed according to a slightly modified protocol of Pitcher and co-workers (Masco et al., 2003; Pitcher et al., 1989). Quality and purity of the obtained DNA extracts were checked spectrophotometrically at 234, 260 and $280 \mathrm{~nm}$ (SpectraMax Plus ${ }^{384}$, Molecular Devices, California, USA) and visually by electrophoresis of $8 \mu \mathrm{L}$ DNA mixed with $2 \mu \mathrm{L}$ loading dye ( $4 \mathrm{~g}$ sucrose and $2.5 \mathrm{mg}$ bromophenol blue dissolved in $6 \mathrm{~mL}$ TE buffer) on a $1 \% \mathrm{w} / \mathrm{v}$ agarose (RESult LE General Purpose Agarose, BIOzym group, Landgraaf, Holland) gel for $30 \mathrm{~min}$ at $100 \mathrm{~V}$ in $1 \times \mathrm{TAE}$ buffer (40 mM Tris-acetate, 1 mM EDTA, pH 8.0), flanked by a molecular weight marker (SmartLadder, Eurogentec, Seraing, Belgium). The procedure for extraction of total DNA from the milk and cheese samples was described previously (Van Hoorde et al., 2008b) and control of the DNA quality and purity was performed as described above.

\subsection{DGGE analysis}

A V3-16S rRNA touchdown PCR (Don et al., 1991), using primers 357f (5'-CCTACGGGAGGCAGCAG-3') coupled to a GC-clamp (5'-CGCCCGCCGCGCGCGGCGGGCGGGGCGGGGGCACGGGGG-3') and 518r (5'-ATTACCGCGGCTGCTGG-3') (Yu and Morrison, 2004), and subsequent Denaturing Gradient Gel Electrophoresis (DGGE) analysis (Temmerman et al., 2003) using a 35 to $60 \%$ denaturing gradient of total cheese DNA and culturable bulk fractions were carried out. The enclosure of a reference pattern every fifth to sixth lane on each DGGE gel allowed to digitally normalize banding patterns by comparing the reference pattern with a standard pattern using BioNumerics software v5.1 (Applied Maths BVBA, Sint-Martens-Latem, Belgium). Normalization permitted comparison of different gels against a DGGEbased identification library generated from band profiles of type and reference strains, identified cheese isolates or extracted DGGE bands tentatively identified by sequencing. Through band position comparison with these database entries, unknown members of the cheese microbiota could be identified.

\subsection{Sequencing of V3-16S rRNA gene amplicons}

DGGE bands which could not be identified by band position analysis using the BioNumerics identification database were extracted from the gel matrix, purified and sequenced as previously described (Van Hoorde et al., 2008b). In addition, a selection of bands that were already assigned to a given species using this database was also included for sequencing in order to confirm identification results obtained by band position analysis. The BLAST algorithm was used to determine the closest relatives in the EMBL nucleotide sequence database.

\subsection{Identification of $L A B$ isolates}

A polyphasic approach combining (GTG) $)_{5}$-PCR profiling and partial $16 \mathrm{~S}$ rRNA and/or phenylalanyl-tRNA synthase (pheS) gene sequencing was used for identification of the 1147 isolates recovered from the four cheeses produced in this study (Van Hoorde et al., 2008a). (GTG) $)_{5}$-PCR was carried out as described previously (Gevers et al., 2001). The resulting (GTG) ${ }_{5}-\mathrm{PCR}$ profiles were compared with an inhouse BioNumerics reference database for identification. Unknown cheese isolates were assigned to a given LAB species when they grouped in a (GTG) $)_{5}$-PCR cluster containing the type strain and one or more reference strains of that species. Isolates that remained unidentified were subsequently subjected to partial 16S rRNA and/ or pheS gene sequencing. To determine closest relatives, partial 16S rRNA gene sequences of unknown isolates were compared with the EMBL sequence database using the BLAST algorithm. pheS sequences were imported into BioNumerics for alignment and comparison with a user-generated database containing pheS sequences of LAB type and reference strains (Scheirlinck et al., 2007).

\subsection{GC-MS profiling}

Cheeses ripened for five weeks were investigated for their volatile composition. A combination of simultaneous steam distillation-extraction (SDE) and gas chromatography-mass spectrometry (GC-MS) was used for the isolation, separation and identification of the cheese volatiles (Van Leuven et al., 2008). For each cheese sample, analysis of the volatile fractions was performed in duplicate. Identification of cheese volatiles was achieved by comparing the mass spectra of the different volatiles to the mass spectra of a commercial Mass Spectral Library, Wiley275 (Wiley, Somerset, NJ, USA). In addition, semi-quantitative information was derived by comparing the relative peak areas of the volatiles to the peak area of an internal undecane standard spiked into the solvent.

\subsection{Data analysis}

To visualize a possible effect of the addition of the two adjunct cultures on the cheese microflora, discriminant analysis (DA) was performed using BioNumerics. In addition, principal component analysis (PCA) was performed on the semi-quantitative GC-MS profiles of the corresponding cheese samples using The Unscrambler 6.1 (Camo, Oslo, Norway). 


\section{Results}

\subsection{Selection of adjunct strains}

From a set of 734 isolates collected and identified by the authors in a previous study (Van Hoorde et al., 2008a), 19 genotypically unique strains were selected as potential adjunct cultures. These included $10 \mathrm{~L}$. paracasei and $4 P$. pentosaceus strains and single strains of $L$. brevis, $L$. curvatus, L. perolens, L. rhamnosus and L. plantarum.

Antibiotic susceptibility testing by disk diffusion revealed that none of the 19 strains exhibited phenotypic resistance to erythromycin (i.e. all inhibition zone diameters $\geq 13 \mathrm{~mm}$ ). In contrast, three strains, i.e. two strains of $P$. pentosaceus and the $L$. curvatus strain, displayed an inhibition zone diameter equal or smaller than $14 \mathrm{~mm}$ for tetracycline thus indicating phenotypic resistance to this antibiotic. This finding was subsequently confirmed by the MIC results $(\geq 16 \mu \mathrm{g} / \mathrm{mL}$, Klare et al., 2007) and by the presence of the corresponding tet(M) resistance gene. These three strains were omitted from further analysis.

The remaining 16 strains were assayed for the presence of proteolytic and lipolytic properties at $14{ }^{\circ} \mathrm{C}$ and $28^{\circ} \mathrm{C}$. None of the strains showed any proteolytic or lipolytic activity on MPCA or TA at $28{ }^{\circ} \mathrm{C}$ except for strain R-49026 belonging to $L$. paracasei which showed proteolytic activity at $28^{\circ} \mathrm{C}$ and which was also the sole strain testing positive for lipase activity next to protease activity at $14^{\circ} \mathrm{C}$. Six other $L$. paracasei strains and one L. perolens strain exhibited protease activity only at $14{ }^{\circ} \mathrm{C}$. Based on the above results, two strains were selected as adjunct culture for the manufacturing of model Gouda-type cheeses. L. paracasei R-49026 was selected because of its pronounced proteolytic and lipolytic properties. As second adjunct culture, L. paracasei $\mathrm{R}-40937$ was selected on the basis of its proteolytic activity at $14{ }^{\circ} \mathrm{C}$ and because it was the only strain which was recovered from the raw milk and during ripening of the corresponding cheese batch produced from this milk (Van Hoorde et al., 2008a).

\subsection{Viable counts of $L A B$}

Four model Gouda-type cheeses with or without adjunct culture were manufactured from a single batch of pasteurized milk. Samples were taken from the curd (C), one-day old cheese (D1) and cheese ripened for 1, 3 and 5 week(s) and plated on the LAB-specific media
KAAA, LM17 and MSE. On all three media, but most explicitly on KAAA, lowest counts were observed for the control cheese G_C (Fig. 1). With respect to the cheeses supplemented with an adjunct culture, no pronounced differences in counts were observed except for the two cheeses supplemented with R-40926 that showed higher overall counts on KAAA medium compared to cheese G_40937. As ripening progressed, all cheeses showed an overall decline in counts on LM17 and MSE. On KAAA, however, counts for the adjunct cheeses remained relatively stable (for G_40937) or even increased (for G_40926) during ripening as opposed to those for G_C which declined. Possibly, these observations can be attributed to the good growth of the respective adjuncts on this particular medium.

\subsection{Identification of $L A B$ isolates}

To monitor the introduction, growth and survival of the adjunct strains in the model cheeses, a total of 1147 randomly selected isolates recovered during the various sampling points on three LAB-specific media were identified and typed using a polyphasic approach. First, the collection of 1147 isolates was dereplicated based on clustering analysis of (GTG) $)_{5}$-PCR fingerprints of crude alkaline extracts. For this purpose, separate dendrograms were constructed for each subset of isolates recovered from a specific cheese at a specific sampling point. By selecting one isolate per (GTG) ${ }_{5}$-cluster, the initial collection was reduced to 130 isolates which were subsequently subjected to a second round of DNA extraction now using a modified version of the method of Pitcher and co-workers (Masco et al., 2003; Pitcher et al., 1989). This method yields DNA extracts that are of superior quality compared to the crude alkaline extracts for generating (GTG) $)_{5}-\mathrm{PCR}$ that need to be compared with the user-generated BioNumerics database for identification. Partial 16S rRNA and/or pheS gene sequencing was performed when isolates could not be unambiguously identified by (GTG) $)_{5}$-PCR profiling. Results of this polyphasic identification approach, including the individual prevalence of the two adjunct strains throughout ripening based on their different (GTG) $)_{5}$-PCR profiles, are shown in Table 1 . In all cheeses, $L$. lactis subsp. lactis and L. pseudomesenteroides were retrieved throughout ripening. Most probably, they originated from the starter as the corresponding (GTG) $)_{5}$-PCR profiles were highly similar to those of L. lactis subsp. lactis and $L$. pseudomesenteroides isolates obtained from the starter. These

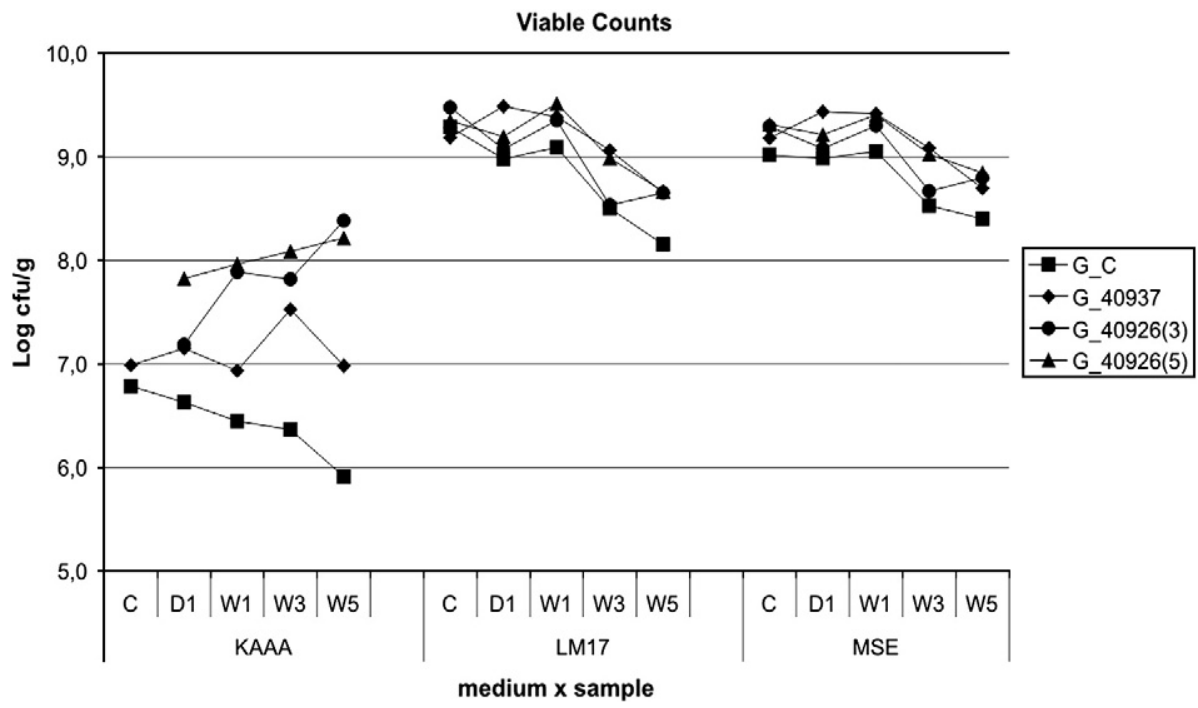

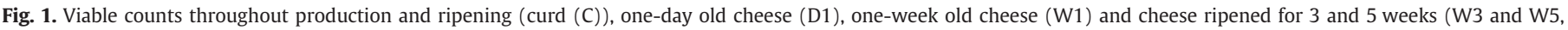

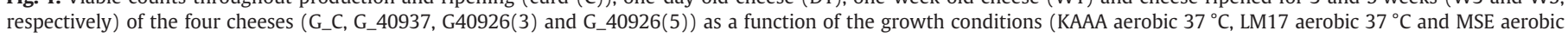
$\left.28{ }^{\circ} \mathrm{C}\right)$. 
Table 1

Biodiversity as determined through isolations and DGGE analysis of total cheese DNA and DNA of culturable fractions.

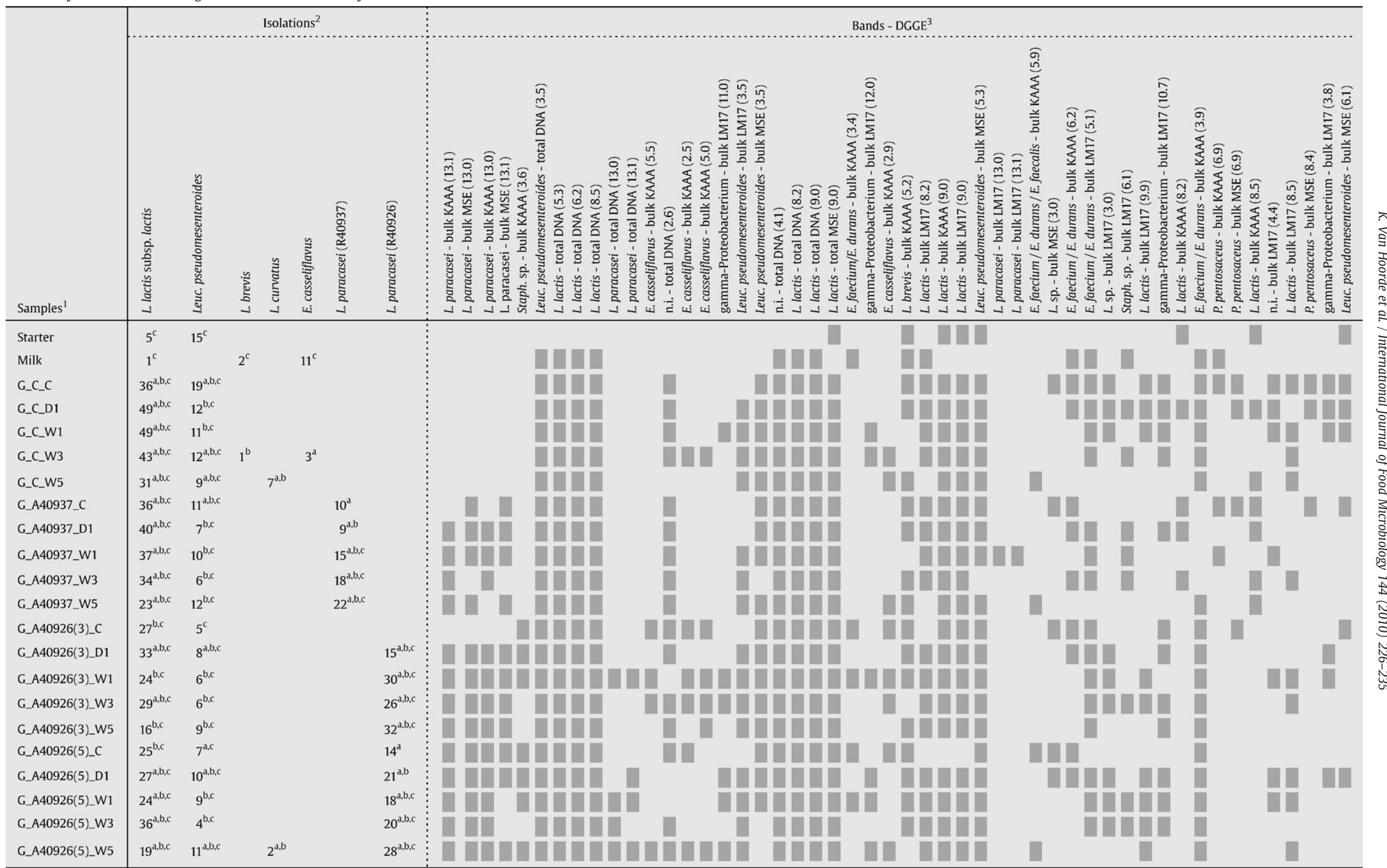

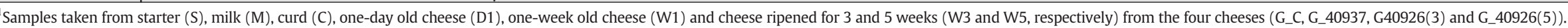
${ }^{2}$ Numbers of isolates with reference to the media they were retrieved from: aKAAA; bLM17; and cMSE.

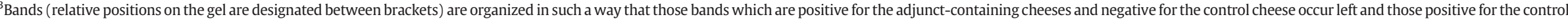
cheese and negative for the adjunct cheeses on the right. 
organisms were dominant in the curd and the early stages of ripening, but their number proportionally decreased as ripening progressed. In the curd and at each phase during ripening, higher numbers of the starter were retrieved in the control cheese compared to the cheeses supplemented with the adjunct strains. No representatives of the two adjunct strains or other strains of $L$. paracasei were retrieved in the control cheese. In cheeses G_40937, G_40926(3) and G_40926 (5), all recovered $L$. paracasei isolates exclusively belonged to the corresponding adjunct cultures used and relative numbers of the adjunct strains increased with ripening. In addition to isolates assigned to the starter or adjunct used, also representatives of L. curvatus, L. brevis and Enterococcus casseliflavus were occasionally found in control and/or adjunct-containing cheeses. The latter two species were also recovered from the milk.

\subsection{PCR-DGGE analysis of total cheese DNA and bulk fractions}

Analysis of DGGE profiles generated from total DNA extracts by band position analysis and band sequencing indicated the presence of members of L. lactis and L. pseudomesenteroides as the predominant species in all four model cheeses. In cheeses G_40926(3) and in particular G_40926(5), the presence of $L$. paracasei was also demonstrated (Table 1). In addition to the aforementioned species, analysis of V3-16S rRNA DGGE profiles of bulk fractions also confirmed the presence of E. casseliflavus, members of the Enterococcus faecium/ Enterococcus durans group, Enterococcus faecalis and P. pentosaceus in various samples of all cheeses. In the DGGE profiles of culturable fractions grown on LM17 medium, bands assigned to Staphylococcus spp. and gamma-Proteobacteria were also found. DGGE profiles generated from the KAAA and MSE fractions clearly demonstrated the presence of $L$. paracasei in the three adjunct cheeses but not in the control cheese (Table 1). Discriminant analysis of the composite dataset encompassing the qualitative and quantitative diversity data of cheese samples obtained by culturing and DGGE allowed to discriminate between control cheese G_C, adjunct cheese G_40937 and the two adjunct cheeses G_40926(3) and G_40926(5) (Fig. 2). Discriminative characters could mainly be attributed to the presence of one or more DGGE bands assigned to L. paracasei and the recovery of isolates representing the two adjunct strains.

\subsection{GC-MS profiling}

The volatile composition of the analyzed Gouda-type cheeses was mainly characterized by aroma compounds derived from lipolysis, proteolysis and metabolism of (residual) lactose, lactate and citrate. The main cheese volatiles could be classified as methylketones, volatile acids, lactones, alcohols, and sulfur compounds (Table 2). The same set of aroma compounds was also previously identified in Gouda-type cheeses (Van Leuven et al., 2008). In this work, some differences were obtained in the volatile composition of the cheese made with adjunct G_40926 compared to the volatile composition of the control cheese sample G_C. In addition, differences were also observed in the amount of aroma compounds originating from lipolysis and proteolysis between cheeses made with the same adjunct R-40926, but in a different final concentration $\left(10^{3}\right.$ and $10^{5} \mathrm{cfu} / \mathrm{mL}$ ). Smaller differences in volatile composition between the control sample and the cheese G_40937 were observed. This was confirmed by the PCA biplot of the volatile fractions analyzed by SDE-GC-MS, which indicated that the control cheese and G_40937 largely exhibited a similar volatile profile. However, the latter two cheeses could clearly be differentiated from the two cheeses made with R-40926 at different concentrations (Fig. 3). These Gouda-type cheeses were clearly characterized by higher amounts of cheese volatiles. Cheese G_40926(3) contained higher levels of compounds derived from proteolysis (2-methylpropanoic acid, 3-methylbutanoic acid and 2-methylbutanoic acid), whereas cheese G_40926(5) was

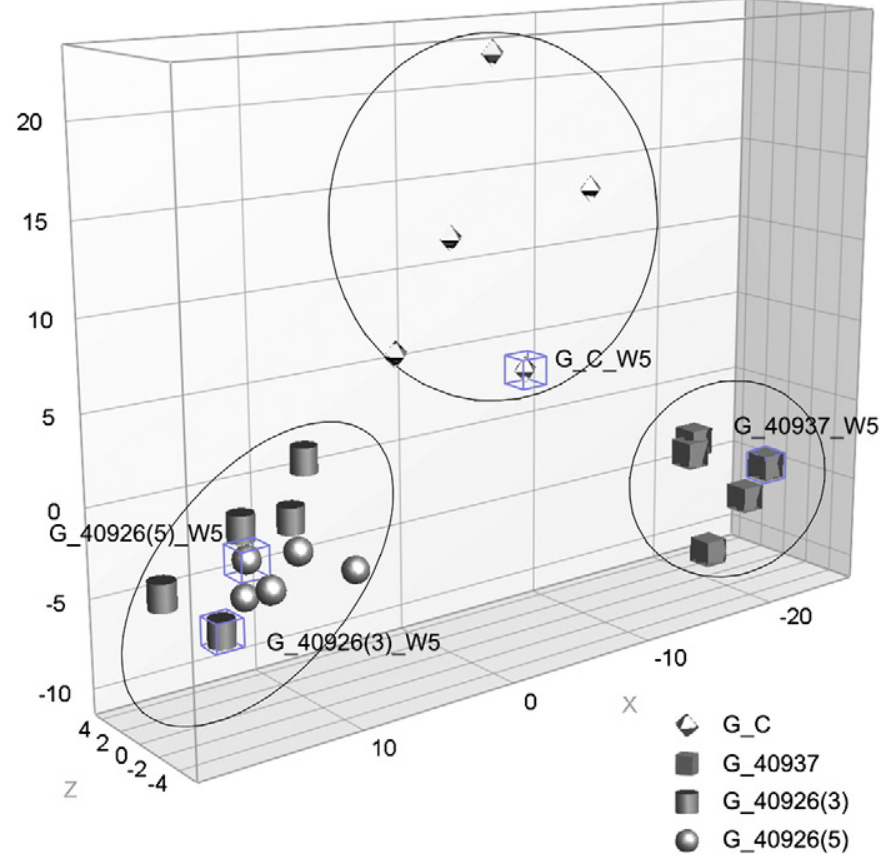

Fig. 2. Tri-dimensional discriminant analysis plot preserving the relative contributions of the three components, i.e. component $1=X=72.8 \%$, component $2=Y=20.8 \%$ and component $3=Z=6.4 \%$. Samples of 5 -week old cheeses (W5) analyzed with SDE-GC-MS are indicated.

dominated by aroma components resulting from both lipolytic (tetradecanoic acid, hexadecanoic acid and lactones) and proteolytic (3-methylbutanal, 2-methylbutanal, 2-phenylethanol and methional) activities.

\section{Discussion}

Several criteria have been put forward for the selection of adjunct strains for use in cheese production. Adjuncts often originate from high-quality aged raw milk cheeses (Poveda et al., 2003; Randazzo et al., 2007), although also non-dairy environments such as sourdough, soil and grass are currently being explored as potential sources of interesting starters or adjuncts for cheese (Ayad et al., 1999; Di Cagno et al., 2006). Next, potential adjunct cultures should be able to reach and maintain high numbers during ripening without affecting the cheese manufacturing process. In addition, technological properties considered important for cheese making such as coagulation and acidifying activities (Randazzo et al., 2007), specific biochemical properties (Tungjaroenchai et al., 2001; van Hylckama Vlieg and Hugenholtz, 2007) and production of bacteriocins (Grattepanche et al., 2008 ) could be taken into account in the process of adjunct screening. Likewise, documentation of safety aspects such as the presence of acquired antibiotic resistance genes and/or potential virulence genes has gained importance in recent years during the selection of LAB strains for food and probiotic applications (Grattepanche et al., 2008). Finally, it is crucial that adjunct candidates should be taxonomically characterized up to the strain level to monitor their persistence and performance during the production of model cheeses.

For the present study, adjunct candidates were selected from an existing collection of 734 isolates previously obtained from two Flemish artisan raw milk Gouda-type cheeses (Van Hoorde et al., 2008a). An initial selection round, in which emphasis was put on strains that are well adapted to the cheese environment by selecting strain types prevalent in both cheese types and/or different production batches analyzed for a given cheese resulted in a total of 19 genotypically unique Lactobacillus and Pediococcus strains. Triggered by increasing concerns regarding the presence and transfer of antibiotic 
Table 2

Volatiles of the control and adjunct cheeses as determined by SDE-GC-MS profiling.

\begin{tabular}{|c|c|c|c|c|}
\hline \multirow[t]{2}{*}{ Aromatic compounds ${ }^{\mathrm{a}, \mathrm{b}, \mathrm{c}}$} & \multicolumn{4}{|l|}{ Cheese } \\
\hline & G_C & G_40973 & G_40926(3) & G_40926(5) \\
\hline \multicolumn{5}{|c|}{ Amino acids } \\
\hline \multicolumn{5}{|c|}{ Aldehydes (branched chain and aromatic) } \\
\hline 3-Methylbutanal & $9.83 \pm 1.10$ & $11.21 \pm 1.43$ & $22.50 \pm 3.14$ & $26.70 \pm 0.76$ \\
\hline 2-Methylbutanal & $1.10 \pm 0.03$ & $1.38 \pm 0.22$ & $1.99 \pm 0.28$ & $2.77 \pm 0.02$ \\
\hline Benzaldehyde & $16.09 \pm 1.52$ & $16.81 \pm 2.96$ & $15.34 \pm 0.71$ & $13.23 \pm 0.00$ \\
\hline Phenylacetaldehyde & $36.03 \pm 1.77$ & $32.04 \pm 1.74$ & $38.81 \pm 0.66$ & $31.85 \pm 0.27$ \\
\hline Sum & $63.04 \pm 2.16$ & $61.43 \pm 0.01$ & $78.63 \pm 2.05$ & $74.54 \pm 0.47$ \\
\hline \multicolumn{5}{|l|}{ Sulfur compounds } \\
\hline Methional & $3.14 \pm 0.32$ & $5.68 \pm 1.15$ & $4.35 \pm 0.26$ & $5.43 \pm 0.03$ \\
\hline \multicolumn{5}{|l|}{ Branched-chain acids } \\
\hline 2-Methylpropanoic acid & & & $8.24 \pm 0.35$ & \\
\hline 3-Methylbutanoic acid & & & $215.60 \pm 31.10$ & \\
\hline 2-Methylbutanoic acid & & & $42.97 \pm 7.04$ & \\
\hline Sum & & & $266.81 \pm 37.79$ & \\
\hline \multicolumn{5}{|l|}{ Alcohols } \\
\hline 1-Butanol & $9.38 \pm 0.94$ & $0.94 \pm 0.45$ & $19.87 \pm 1.51$ & $21.88 \pm 0.23$ \\
\hline 3-Methyl-1-butanol & $3.94 \pm 0.40$ & $10.67 \pm 0.01$ & $35.15 \pm 6.99$ & $26.91 \pm 1.27$ \\
\hline 2-Methyl-1-butanol & n.q. & n.q. & $12.90 \pm 3.10$ & $11.44 \pm 0.85$ \\
\hline 2-Phenylethanol & $2.53 \pm 0.56$ & $13.41 \pm 0.03$ & $4.62 \pm 0.01$ & $11.40 \pm 1.20$ \\
\hline Sum & $15.84 \pm 1.90$ & $34.04 \pm 0.49$ & $72.52 \pm 11.61$ & $71.63 \pm 1.15$ \\
\hline 1H-indole & $6.93 \pm 0.41$ & $5.09 \pm 0.64$ & $4.57 \pm 0.05$ & $2.88 \pm 0.11$ \\
\hline \multicolumn{5}{|l|}{ Carbohydrates } \\
\hline \multicolumn{5}{|l|}{ Ketones } \\
\hline 3-Hydroxy-2-butanone & $653.68 \pm 97.46$ & $215.50 \pm 3.18$ & $546.67 \pm 154.65$ & $358.88 \pm 79.01$ \\
\hline 2,3-Pentanedione & $4.66 \pm 0.45$ & $8.08 \pm 2.93$ & $4.97 \pm 0.06$ & $4.88 \pm 0.49$ \\
\hline Sum & $658.34 \pm 97.91$ & $223.58 \pm 0.25$ & $551.63 \pm 154.71$ & $363.76 \pm 79.50$ \\
\hline Alcohols & & & & \\
\hline 2,3-Butanediol & $23.09 \pm 4.78$ & $36.80 \pm 7.25$ & $21.44 \pm 6.31$ & $28.19 \pm 7.22$ \\
\hline Other & & & & \\
\hline Furfurol & $70.96 \pm 3.32$ & $52.85 \pm 2.67$ & $65.60 \pm 4.41$ & $54.43 \pm 0.44$ \\
\hline 2-Pentylfurane & $12.43 \pm 0.04$ & $6.51 \pm 0.97$ & $9.99 \pm 1.46$ & $7.06 \pm 0.30$ \\
\hline$\alpha$-Pinene & $1.58 \pm 0.08$ & 2.77 & $3.76 \pm 0.18$ & $2.83 \pm 0.16$ \\
\hline Limonene & $6.84 \pm 0.44$ & $6.93 \pm 0.37$ & $7.48 \pm 0.30$ & $6.39 \pm 0.35$ \\
\hline 9-Decene-2-on & $29.80 \pm 5.61$ & $27.25 \pm 0.48$ & $27.65 \pm 0.06$ & $30.29 \pm 1.97$ \\
\hline Sum & $121.60 \pm 9.25$ & $94.92 \pm 6.44$ & $114.47 \pm 6.43$ & $101.00 \pm 1.75$ \\
\hline Lipids & & & & \\
\hline Linear aldehydes & & & & \\
\hline Pentanal & $3.88 \pm 0.32$ & $3.21 \pm 0.22$ & $3.89 \pm 0.34$ & $3.35 \pm 0.02$ \\
\hline Hexanal & $25.18 \pm 0.87$ & $0.87 \pm 2.02$ & $24.15 \pm 1.87$ & $13.27 \pm 2.09$ \\
\hline Heptanal & $5.77 \pm 0.74$ & $4.71 \pm 0.64$ & $6.18 \pm 0.62$ & $6.56 \pm 0.95$ \\
\hline Nonanal & $35.11 \pm 7.77$ & $25.60 \pm 3.06$ & $35.55 \pm 3.62$ & $36.99 \pm 4.51$ \\
\hline Decanal & $7.62 \pm 1.82$ & $3.15 \pm 0.23$ & $3.61 \pm 0.54$ & $3.93 \pm 0.47$ \\
\hline Dodecanal & $33.49 \pm 3.71$ & $32.08 \pm 3.34$ & $27.28 \pm 0.84$ & $26.55 \pm 1.24$ \\
\hline Tetradecanal & $149.36 \pm 10.06$ & $122.11 \pm 20.32$ & $113.88 \pm 14.03$ & $112.96 \pm 0.72$ \\
\hline t,t-2,4-Decadienal & $9.27 \pm 0.95$ & $8.26 \pm 2.38$ & $8.02 \pm 0.64$ & $7.41 \pm 1.15$ \\
\hline 2-Octenal & $3.54 \pm 0.30$ & $2.61 \pm 0.21$ & $3.34 \pm 0.11$ & $2.73 \pm 0.27$ \\
\hline 2-Nonenal & $6.27 \pm 1.12$ & $4.50 \pm 0.93$ & $5.75 \pm 0.50$ & $5.81 \pm 0.99$ \\
\hline 2-Decenal & $21.13 \pm 3.49$ & n.q. & $19.14 \pm 2.97$ & $15.91 \pm 2.31$ \\
\hline Sum & $300.59 \pm 29.41$ & $219.25 \pm 26.66$ & $250.77 \pm 11.96$ & $235.45 \pm 13.28$ \\
\hline Methylketones & & & & \\
\hline 2-Pentanone & $628.41 \pm 17.00$ & $631.04 \pm 0.18$ & $692.72 \pm 18.09$ & $637.13 \pm 44.80$ \\
\hline 2-Hexanone & $37.64 \pm 3.11$ & $35.92 \pm 0.10$ & $40.36 \pm 2.72$ & $36.93 \pm 3.89$ \\
\hline 2-Heptanone & $2063.03 \pm 103.22$ & $2003.05 \pm 16.18$ & $2133.17 \pm 40.60$ & $2032.60 \pm 107.32$ \\
\hline 2-Octanone & $36.60 \pm 2.64$ & $29.49 \pm 0.25$ & $34.64 \pm 4.62$ & $31.36 \pm 1.88$ \\
\hline 2-Nonanone & $1701.42 \pm 183.95$ & $1580.55 \pm 11.58$ & $1653.32 \pm 38.58$ & $1675.30 \pm 14.09$ \\
\hline 2-Decanone & $32.51 \pm 4.35$ & $28.16 \pm 0.83$ & $30.65 \pm 0.31$ & $30.91 \pm 1.55$ \\
\hline 2-Undecanone & $1999.46 \pm 327.36$ & $1881.30 \pm 21.86$ & $1783.03 \pm 29.90$ & $2030.46 \pm 7.50$ \\
\hline 2-Dodecanone & $58.28 \pm 5.81$ & $49.09 \pm 1.93$ & $50.15 \pm 3.54$ & $62.33 \pm 2.42$ \\
\hline 2-Tridecanone & $2367.49 \pm 431.61$ & $2212.59 \pm 147.85$ & $1956.87 \pm 139.95$ & $2412.66 \pm 43.92$ \\
\hline 2-Tetradecanone & $59.68 \pm 10.54$ & $55.32 \pm 5.28$ & $49.50 \pm 4.29$ & $59.66 \pm 0.60$ \\
\hline 2-Pentadecanone & $2684.50 \pm 395.20$ & $2489.95 \pm 319.45$ & $2103.60 \pm 313.50$ & $2575.06 \pm 108.14$ \\
\hline Sum & $11,669.01 \pm 1479.51$ & $10,996.44 \pm 469.12$ & $10,527.99 \pm 386.87$ & $11,584.38 \pm 31.99$ \\
\hline Secondary alcohols & & & & \\
\hline 2-Butanol & $11.98 \pm 1.21$ & $33.27 \pm 0.59$ & $12.65 \pm 0.77$ & $26.81 \pm 3.30$ \\
\hline 2-Pentanol & $10.82 \pm 0.50$ & $41.79 \pm 7.32$ & $33.14 \pm 6.93$ & $31.52 \pm 2.72$ \\
\hline 2-Heptanol & $5.71 \pm 0.50$ & $14.42 \pm 0.48$ & $13.62 \pm 2.21$ & $14.73 \pm 1.72$ \\
\hline Sum & $28.50 \pm 0.21$ & $89.48 \pm 7.20$ & $59.41 \pm 3.95$ & $73.05 \pm 2.29$ \\
\hline Volatile fatty acids & 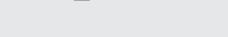 & & & \\
\hline Butanoic acid & $60.79 \pm 24.37$ & & $235.01 \pm 42.09$ & \\
\hline Hexanoic acid & $261.64 \pm 14.57$ & $16.84 \pm 4.16$ & $528.25 \pm 18.70$ & \\
\hline Octanoic acid & $914.58 \pm 214.76$ & $296.38 \pm 119.93$ & $1422.71 \pm 71.40$ & $65.70 \pm 2.64$ \\
\hline Volatile fatty acids & & & & \\
\hline Decanoic acid & $5857.36 \pm 1648.86$ & $5168.54 \pm 1039.81$ & $6843.04 \pm 113.21$ & $3851.88 \pm 193.92$ \\
\hline Undecanoic acid & $51.69 \pm 16.10$ & $51.57 \pm 15.81$ & $67.58 \pm 0.01$ & $47.06 \pm 2.14$ \\
\hline
\end{tabular}


Table 2 (continued)

\begin{tabular}{|c|c|c|c|c|}
\hline \multirow[t]{2}{*}{ Aromatic compounds ${ }^{\mathrm{a}, \mathrm{b}, \mathrm{c}}$} & \multicolumn{4}{|l|}{ Cheese } \\
\hline & G_C & G_40973 & G_40926(3) & G_40926(5) \\
\hline Dodecanoic acid & $4609.45 \pm 1144.49$ & $4525.62 \pm 1052.02$ & $4814.57 \pm 309.89$ & $4717.04 \pm 87.48$ \\
\hline Tetradecanoic acid & $2297.62 \pm 535.33$ & $2218.97 \pm 706.39$ & $2135.27 \pm 359.37$ & $2514.16 \pm 10.87$ \\
\hline Hexadecanoic acid & $594.36 \pm 90.17$ & $538.33 \pm 173.33$ & $549.98 \pm 123.48$ & $648.59 \pm 17.06$ \\
\hline Sum & $14,647.47 \pm 3610.76$ & $12,816.25 \pm 3111.45$ & $16,596.38 \pm 857.95$ & $11,844.42 \pm 309.83$ \\
\hline \multicolumn{5}{|l|}{ Lactones } \\
\hline$\gamma$-Nonalactone & $6.42 \pm 0.79$ & $5.81 \pm 0.49$ & $6.22 \pm 0.13$ & $6.07 \pm 0.13$ \\
\hline$\gamma$-Decalactone & $16.33 \pm 1.75$ & $14.78 \pm 1.80$ & $14.28 \pm 0.69$ & $17.01 \pm 0.44$ \\
\hline Cis- $\gamma$-6-dodecenoic acid lactone & $340.63 \pm 49.13$ & $297.17 \pm 48.48$ & $290.98 \pm 35.60$ & $356.52 \pm 11.28$ \\
\hline$\gamma$-Dodecalactone & $962.90 \pm 139.36$ & $841.11 \pm 119.68$ & $804.39 \pm 98.34$ & $985.29 \pm 29.80$ \\
\hline$\delta$-Hexalactone & $7.81 \pm 0.79$ & $7.60 \pm 1.79$ & $8.08 \pm 1.72$ & $7.83 \pm 1.40$ \\
\hline$\delta$-Octalactone & $56.80 \pm 5.23$ & $49.79 \pm 8.00$ & $57.52 \pm 4.04$ & $56.99 \pm 1.55$ \\
\hline$\delta$-Decalactone & $1131.17 \pm 134.99$ & $982.21 \pm 160.34$ & $997.62 \pm 107.70$ & $1127.52 \pm 35.26$ \\
\hline$\delta$-Undecalactone & $43.29 \pm 7.09$ & $38.16 \pm 7.35$ & $36.74 \pm 5.14$ & $44.10 \pm 2.64$ \\
\hline$\delta$-Dodecalactone & $1709.16 \pm 213.56$ & $1473.75 \pm 245.69$ & $1377.20 \pm 225.88$ & $1633.40 \pm 97.16$ \\
\hline$\delta$-Tetradecalactone & $719.68 \pm 56.02$ & $597.26 \pm 87.24$ & $540.82 \pm 122.41$ & $642.53 \pm 33.96$ \\
\hline$\delta$-Hexadecalactone & $102.80 \pm 0.96$ & $92.63 \pm 25.29$ & $85.36 \pm 19.93$ & $101.17 \pm 9.16$ \\
\hline Sum & $5096.97 \pm 608.08$ & $4400.27 \pm 706.17$ & $4219.19 \pm 621.31$ & $4978.41 \pm 222.51$ \\
\hline Total sum & $32,634.49 \pm 5844.29$ & $28,983.18 \pm 4319.11$ & $32,768.12 \pm 1757.93$ & $29,363.12 \pm 594.61$ \\
\hline
\end{tabular}

a Average and standard deviation of semi-quantitative values of cheese aromatic compounds after duplicate analysis of the volatile fractions.

b n.q.: not quantified.

c Zero measurements are reported as blank cells.

resistance in food-related LAB (Ammor et al., 2007) and the observation that antibiotic resistance in LAB strains is most commonly linked to tetracycline and/or erythromycin resistance (Klare et al., 2007; Van Hoek et al., 2008), representative isolates of these 19 strain types were tested for phenotypic resistance to these two antibiotics. Two strains of $P$. pentosaceus and a $L$. curvatus strain exhibited phenotypic resistance against tetracycline, which in all three cases could be linked to the presence of the ribosomal protection gene tet $(\mathrm{M})$. Although the genomic location and the potential transferability of this gene were not further investigated, it was decided at this stage of the study to omit the three tetracycline-resistant strains from the selection of adjunct candidates as a precautionary measure. The fact that the tet $(\mathrm{M})$ gene is integrated in members of the broad host range family of Tn916-1545 conjugative transposons (Hummel et al., 2007) could pose a potential risk for horizontal transfer of tetracycline resistance between members of the cheese ecosystem. Previously, tet( $\mathrm{M})$ genes have been found in
L. paracasei either with (Devirgiliis et al., 2009) or without (Huys et al., 2008) the presence of Tn916.

Evaluation of protein- and fat-degrading capacities was evaluated for the 16 remaining strains devoid of the tested antibiotic resistances which resulted in a final set of two $L$. paracasei strains with potential adjunct properties, R-40926 and R-40937. The latter strain was recovered from raw milk which is considered an important source of NSLAB with potential adjunct properties (Franciosi et al., 2009). In order to monitor the introduction of these two selected adjunct strains in the cheese making process and their effect on the cheese microbiota during ripening, a combination of culture-dependent and independent methods was used. Selective isolations on three LAB-specific media and (GTG) $)_{5}$-PCR profiling revealed that members of the starter, i.e. L. lactis subsp. lactis and L. pseudomesenteroides, were dominant in all production stages of the control cheese as well as in the early stages of ripening of the adjunct-containing cheeses. This finding concurred

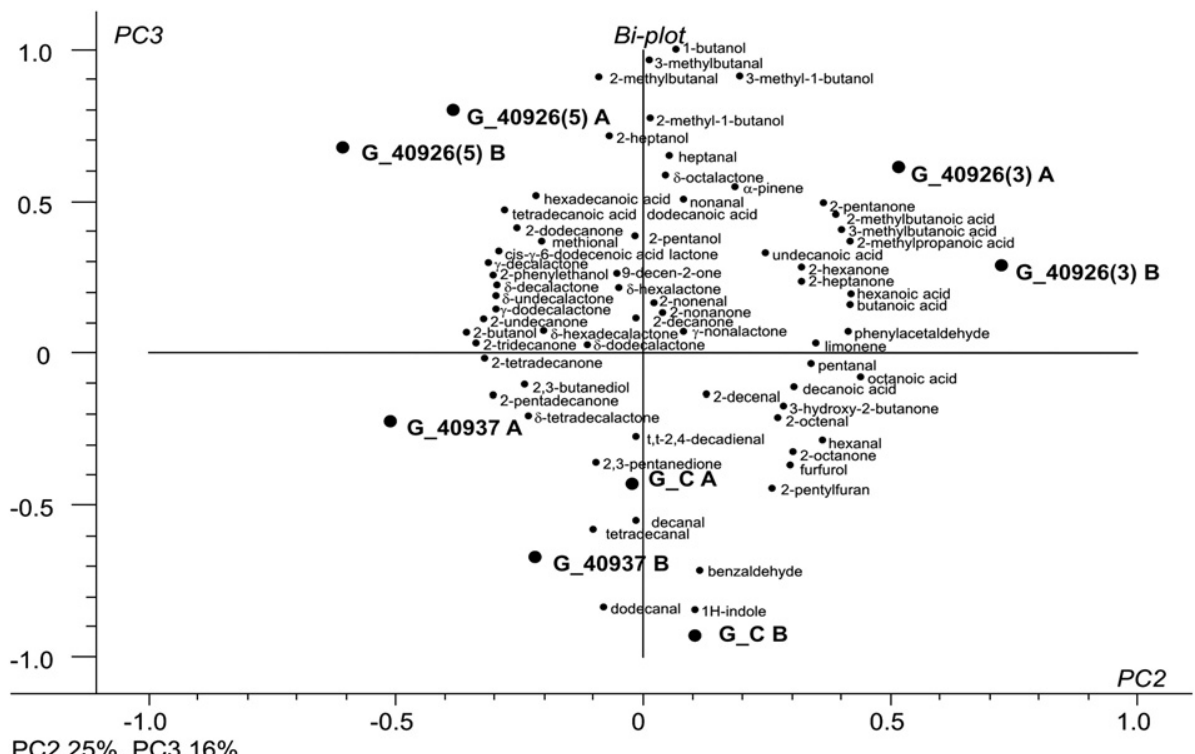

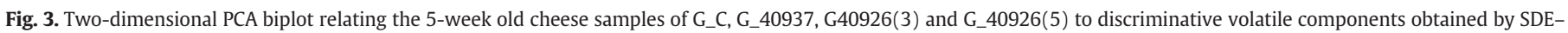
GC-MS. A and B correspond with the duplicate GC-MS analyses. 
with the results from culture-independent analysis which clearly showed the presence of predominant bands assigned to members of the starter in the DGGE profiles of total cheese DNA extracts in all four cheeses. (GTG) 5 $_{5}$-PCR profiling of isolates from cheeses G_40937 and G_40926(3)/(5) confirmed the presence of adjunct strains L. paracasei R-40926 and R-40937 in the cheeses in which they were respectively used. The observation that their relative numbers increased as ripening progressed indicates that both strains are well adapted to the cheese environment and can survive in a competitive environment in the presence of a commercial starter culture. DGGE analysis obtained from total DNA extracts of the adjunct-containing cheeses revealed the presence of $L$. paracasei in only a few samples, whereas DGGE profiles of the corresponding culturable fractions, especially those grown on KAAA and MSE, clearly demonstrated the presence of $L$. paracasei. Possibly, preferential amplification and differences in amplification efficiency (Suzuki and Giovannoni, 1996) or the possibility that some of the media used clearly favored growth of $L$. paracasei could explain these differences between culture-dependent and culture-independent approaches. Although overall differences in diversity were minimal, the control cheese could be differentiated from the adjunct-containing cheeses by discriminant analysis on the basis of the absence or presence of isolates and bands assigned to $L$. paracasei. Moreover, cheeses G_40937 and G_40926(3)/(5) could also be discriminated based on differences in the (GTG) ${ }_{5}$-profiles of the corresponding $L$. paracasei adjunct isolates. For cheeses supplemented with strain R-40926, no concentration effect on the overall diversity could be demonstrated. To verify whether this is due to a normalization of both concentrations during cheese production or the absence of a true concentration effect, absolute numbers of the adjuncts in the cheeses would need to be determined e.g. by using species- or strain-specific primers in a real-time PCR assay.

In previous studies, the use of $L$. paracasei strains as adjuncts and its different effects on the volatile composition of the ripened cheese have been documented. For Danish Danbo cheeses, it has been shown that either beneficial effects or off-flavours were obtained depending on the L. paracasei adjunct strain added (Antonsson et al., 2003). Lynch et al. (1999) investigated the influence of amongst others L. paracasei subsp. paracasei on the ripening of Cheddar cheeses and observed an increase in flavour intensity and bitter flavour whereas the creaminess and milky flavour decreased. Probiotic cheeses made with $L$. paracasei were perceived significantly different from the probiotic free cheeses, with higher scores for bitterness (Ong et al., 2007). In the present study, a descriptive sensory analysis of the four cheeses according to the method previously described by Van Leuven et al. (2008) revealed that the scores for the descriptor 'aroma intensity' increased in the cheese to which adjunct culture R-40926, irrespective of the concentration, was added (ANOVA: $0.05<P<0.10$; data not shown). Furthermore, PCA of the volatile compounds could clearly differentiate cheeses made with adjunct R-40926, in particular G_40926(3), from the control cheese G_C which showed only small differences with the cheese made with adjunct R-40937. The cheese made with adjunct R-40926(3) was mainly characterized by volatiles derived from proteolysis (branched-chain volatile acids), while the cheese made with adjunct R-40926(5) was dominated by fat-related compounds such as free fatty acids and $\gamma$ - and $\delta$-lactones, of which the concentration generally correlates with the extend of lipolysis (Collins et al., 2003), next to some amino acid-related compounds (branched-chain aldehydes and sulfur compounds). These results are in line with the observed technological properties of R-40926 which showed next to proteolytic also lipolytic properties, whereas R-40937 only showed evidence of some proteolytic activity on milk plate count agar.

The use of adjunct cultures in the production of new types of artisan Gouda-type cheeses is a promising strategy to compensate for flavour uniformities due to a loss of biodiversity in the cheeses. In this study, we propose one possible approach to screen well-characterized NSLAB with clear predominance in raw milk cheeses for basic safety and technological parameters with the aim of using a subset of selected cultures as adjuncts in model cheese production. Although outside the scope of this study, additional functional screening for the presence of specific flavour-forming enzymes and their metabolic products could further contribute to this selection process. Together with a better understanding of the population dynamics with respect to interactions of the introduced adjuncts with other bacteria predominating the cheese ecosystem, this strategy could pave the way to diversify the current range of Gouda-type and other semi-hard type cheeses by enriching or extending their flavour palette.

\section{Acknowledgements}

This research was supported by the Institute for the Promotion of Innovation through Science and Technology in Flanders (IWTVlaanderen). The fund for Scientific Research-Flanders (FWO-Vlaanderen) is acknowledged for the postdoctoral fellowship of Geert Huys. We thank Willy Deville for his technical advice and assistance. We are also grateful to the cheese manufacture workers for their cooperation, for the use of their equipment and for providing the raw material and samples used in this study.

\section{References}

Ammor, M.S., Belén, F.A., Mayo, B., 2007. Antibiotic resistance in non-enterococcal lactic acid bacteria and bifidobacteria. Food Microbiology 24, 559-570.

Antonsson, M., Molin, G., Ardö, Y., 2003. Lactobacillus strains isolated from Danbo cheese as adjunct cultures in a cheese model system. International Journal of Food Microbiology 85, 159-169.

Ayad, E.H.E., Verheul, A., de Jong, C., Wouters, J.T.M., Smit, G., 1999. Flavour forming abilities and amino acid requirements of Lactococcus lactis strains isolated from artisanal and non-dairy origin. Internatinoal Dairy Journal 9, 725-735.

Broadbent, J.R., Steele, J.L., 2005. Cheese flavor and the genomics of lactic acid bacteria ASM News-American Society for Microbiology 71, 121-128.

Bauer, A.W., Kirby, W.M.M., Sherris, J.C., Turck, M., 1966. Antibiotic susceptibility testing by a standardized single disk method. American Journal of Clinical Pathology 45, 493-496.

Buchin, S., Delague, V., Duboz, G., Berdague, J.L., Beuvier, E., Pochet, S., Grappin, R., 1998. Influence of pasteurization and fat composition of milk on the volatile compounds and flavor characteristics of a semi-hard cheese. Journal of Dairy Science 81, 3097-3108.

Charteris, W.P., Kelly, P.M., Morelli, L., Collins, J.K., 1998. Antibiotic susceptibility of potential probiotic Lactobacillus species. Journal of Food Protection 61, 1636-1643.

Collins, Y.F., McSweeney, P.L.H., Wilkinson, M.G., 2003. Lipolysis and free fatty acid catabolism in cheese: a review of current knowledge. International Dairy Journal 13, 841-866.

Devirgiliis, C., Coppola, D., Barile, S., Colonna, B., Perozzi, G., 2009. Characterization of the Tn916 conjugative transposon in a foodborne strain of Lactobacillus paracasei. Applied and Environmental Microbiology 75, 3866-3871.

Di Cagno, R., Quinto, M., Corsetti, A., Minervini, F., Gobbetti, M., 2006. Assessing the proteolytic and lipolytic activities of single strains of mesophilic lactobacilli as adjunct cultures using a Caciotta cheese model system. International Dairy Journal 16, 119-130.

Don, R.H., Cox, P.T., Wainwright, B.J., Baker, K., Mattick, J.S., 1991. 'Touchdown' PCR to circumvent spurious priming during gene amplification. Nucleic Acids Research 19, 4008.

Ercolini, D., Moschetti, G., Blaiotta, G., Coppola, S., 2001. The potential of a polyphasic PCR-DGGE approach in evaluating microbial diversity of natural whey cultures for water-buffalo Mozzarella cheese production: bias of culture-dependent and culture-independent analyses. Systematic and Applied Microbiology 24, 610-617.

Fox, P.F., McSweeney, P.L.H., Lynch, C.M., 1998. Significance of non-starter lactic acid bacteria in Cheddar cheese. Australian Journal of Dairy Technology 53, 83-89.

Franciosi, E., Settanni, L., Cavazza, A., Poznanski, E., 2009. Biodiversity and technological potential of wild lactic acid bacteria from raw cows' milk. International Dairy Journal 19, 3-11.

Gevers, D., Huys, G., Swings, J., 2001. Applicability of rep-PCR fingerprinting for identification of Lactobacillus species. FEMS Microbiology Letters 205, 31-36.

Gómez-Ruiz, J., Cabezas, L., Martínez-Castro, I., González-Viñas, M., Poveda, J., 2008 Influence of a defined-strain starter and Lactobacillus plantarum as adjunct culture on volatile compounds and sensory characteristics of Manchego cheese. European Food Research and Technology 227, 181-190.

Grappin, R., Beuvier, E., 1997. Possible implications of milk pasteurization on the manufacture and sensory quality of ripened cheese - proteolytic enzymes and cheese ripening - proteolytic enzymes and their relation to cheese ripening and flavor: an overview. International Dairy Journal 7, 751-761.

Grattepanche, F., Miescher-Schwenninger, S., Meile, L., Lacroix, C., 2008. Recent developments in cheese cultures with protective and probiotic functionalities. Dairy Science and Technology 88, 421-444.

Herreros, M.A., Arenas, R., Sandoval, M.H., Castro, J.M., Fresno, J.M., Tornadijo, M.E. 2007. Effect of addition of native cultures on characteristics of Armada cheese 
manufactured with pasteurized milk: a preliminary study. Internatinoal Dairy Journal 17, 328-335.

Hummel, A., Holzapfel, W.H., Franz, C.M., 2007. Characterisation and transfer of antibiotic resistance genes from enterococci isolated from food. Systematic and Applied Microbiology 30, 1-7.

Huys, G., D'Haene, K., Danielsen, M., Mättö, J., Egervärn, M., Vandamme, P., 2008. Phenotypic and molecular assessment of antimicrobial resistance in Lactobacillus paracasei strains from food origin. Journal of Food Protection 71, 339-344.

Kieronczyk, A., Skeie, S., Langsrud, T., Yvon, M., 2003. Cooperation between Lactococcus lactis and nonstarter lactobacilli in the formation of cheese aroma from amino acids. Applied and Environmental Microbiology 69, 734-739.

Klare, I., Konstabel, C., Muller-Bertling, S., Reissbrodt, R., Huys, G., Vancanneyt, M. Swings, J., Goossens, H., Witte, W., 2005. Evaluation of new broth media for microdilution antibiotic susceptibility testing of lactobacilli, pediococci, lactococci, and bifidobacteria. Applied and Environmental Microbiology 71, 8982-8986.

Klare, I., Konstabel, C., Werner, G., Huys, G., Vankerckhoven, V., Kahlmeter, G., Hildebrandt, B., Muller-Bertling, S., Witte, W., Goossens, H., 2007. Antimicrobial susceptibilities of Lactobacillus, Pediococcus and Lactococcus human isolates and cultures intended for probiotic or nutritional use. Journalof Antimicrobia Chemotherapy 59, 900-912.

Lee, B.H., Laleye, L.C., Simard, R.E., Holley, R.A., Emmons, D.B., Giroux, R.N., 1990 Influence of homofermentative lactobacilli on physicochemical and sensory properties of cheddar cheeseInfluence of homofermentative lactobacilli on physicochemical and sensory properties of cheddar cheese. Journal of Food Science 55, 386-390

Lynch, C.M., McSweeney, P.L.H., Fox, P.F., Cogan, T.M., Drinan, F.D., 1996. Manufacture of Cheddar cheese with and without adjunct lactobacilli under controlled microbiological conditions. Interational Dairy Journal 6, 851-867.

Lynch, C.M., Muir, D.D., Banks, J.M., McSweeney, P.L.H., Fox, P.F. 1999. Influence of adjunct cultures of Lactobacillus paracasei ssp. paracasei or Lactobacillus plantarum on Cheddar cheese ripening. Journal of Dairy Science 82, 1618-1628.

Marilley, L., Casey, M.G., 2004. Flavours of cheese products: metabolic pathways, analytical tools and identification of producing strains. International Journal of Food Microbiology 90, 139-159.

Masco, L., Huys, G., Gevers, D., Verbrugghen, L., Swings, J., 2003. Identification of Bifidobacterium species using rep-PCR fingerprinting. Systematic and Applied Microbiology 26, 557-563.

Masco, L., Van Hoorde, K., De Brandt, E., Swings, J., Huys, G., 2006. Antimicrobial susceptibility of Bifidobacterium strains from humans, animals and probiotic products. The Journal of Antimicrobial Chemotherapy 58, 85-94.

McSweeney, P.L.H., 2004. Biochemistry of cheese ripening. International Journal of Dairy Technology 57, 127-144.

Ong L, Henriksson, A., Shah, N.P., 2007. Chemical analysis and sensory evaluation of Cheddar cheese produced with Lactobacillus acidophilus, Lb. casei, Lb. paracasei or Bifidobacterium sp. International Dairy Journal 17, 937-945.

Pitcher, D.G., Saunders, N.A., Owen, R.J., 1989. Rapid extraction of bacterial genomic DNA with guanidium thiocyanate. Letters in Applied Microbiology 8, 151-156.
Poveda, J.M., Sousa, M.J., Cabezas, L., McSweeney, P.L.H., 2003. Preliminary observations on proteolysis in Manchego cheese made with a defined-strain starter culture and adjunct starter (Lactobacillus plantarum) or a commercial starter. International Dairy Journal 13, 169-178.

Randazzo, C.L., De Luca, S., Todaro, A., Restuccia, C., Lanza, C.M., Spagna, G., Caggia, C., 2007. Preliminary characterization of wild lactic acid bacteria and their abilities to produce flavour compounds in ripened model cheese system. Journal of Applied Microbiology 103, 427-435.

Scheirlinck, I., Van der Meulen, R., Van Schoor, A., Vancanneyt, M., De Vuyst, L., Vandamme, P., Huys, G., 2007. Influence of geographical origin and flour type on diversity of lactic acid bacteria in traditional Belgian sourdoughs. Applied and Environmental Microbiology 7319, 6262-6269.

Smit, G., Smit, B.A., Engels, W.J.M., 2005. Flavour formation by lactic acid bacteria and biochemical flavour profiling of cheese products. FEMS Microbiology Reviews 29, 591-610.

Suzuki, M.T., Giovannoni, S.J., 1996. Bias caused by template annealing in the amplification of mixtures of 16S rRNA genes by PCR. Applied and Environmental Microbiology 62, 625-630.

Temmerman, R., Scheirlinck, I., Huys, G., Swings, J., 2003. Culture-independent analysis of probiotic products by denaturing gradient gel electrophoresis. Applied and Environmental Microbiology 69, 220-226.

Tungjaroenchai, W., Drake, M.A., White, C.H., 2001. Influence of adjunct cultures on ripening of reduced fat Edam cheeses. Journal of Dairy Science 84, 2117-2124.

van den Berg, G., 2003. Cheeses/Dutch-type cheeses. In: Caballero, B., Trugo, L., Finglas, P.M. (Eds.), Encyclopedia of Food Sciences and Nutrition. Academic Press, Oxford, pp. $1129-1135$

van Hoek, A.H.A.M., Margolles, A., Domig, K.J., Korhonen, J.M., Zycka-Krzesinska, J., Bardowski, J.K., Danielsen, M., Huys, G., Morelli, L., Aarts, H.J.M., 2008. Molecular assessment of erythromycin and tetracycline resistance genes in lactic acid bacteria and bifidobacteria and their relation to the phenotypic resistance. International Journal of Probiotics and Prebiotics 3, 271-280.

Van Hoorde, K., Vandamme, P., Huys, G., 2008a. Molecular identification and typing of lactic acid bacteria associated with the production of two artisanal raw milk cheeses. Dairy Science and Technology 88, 445-455.

Van Hoorde, K., Verstraete, T., Vandamme, P., Huys, G., 2008b. Diversity of lactic acid bacteria in two Flemish artisan Gouda-type cheeses. Food Microbiology 25, 929-935.

van Hylckama Vlieg, J.E.T., Hugenholtz, J., 2007. Mining natural diversity of lactic acid bacteria for flavour and health benefits. International Dairy Journal 17, 1290-1297.

Van Leuven, I., Van Caelenberg, T., Dirinck, P., 2008. Aroma characterisation of Gouda-type cheeses. International Dairy Journal 18, 790-800.

Wouters, J.T.M., Ayad, E.H.E., Hugenholtz, J., Smit, G., 2002. Microbes from raw milk for fermented dairy products. International Dairy Journal 12, 91-109.

$\mathrm{Yu}, \mathrm{Z}$, Morrison, M., 2004. Comparisons of different hypervariable regions of rrs genes for use in fingerprinting of microbial communities by PCR-denaturing gradient gel electrophoresis. Applied and Environmental Microbiology 70, 4800-4806. 